\title{
Estudo comparativo da metodologia ativa "gincana" nas modalidades presencial e à distância em curso de graduação de Farmácia
}

\author{
Comparative study of "scavenger hunt" active methodology between \\ face-to-face and distance learning in Pharmacy undergraduate course
}

Agnes Nogueira Gossenheimer ${ }^{1}$, Mára Lúcia Fernandes Carneiro², Mauro Silveira de Castro

${ }^{1}$ Faculdade de Farmácia da Universidade Federal do Rio Grande do Sul (UFRGS) - Porto Alegre (RS), Brasil.

${ }^{2}$ Instituto de Psicologia, UFRGS - Porto Alegre (RS), Brasil.

DOI: http://dx.doi.org/10.7322/abcshs.v40i3.801

\section{RESUMO}

Introdução: O crescimento da educação a distância (EaD) no Brasil tem sido cada vez mais intenso, inclusive em cursos da saúde. Objetivos: Realizar análise comparativa da "Gincana" nas modalidades presencial e a distância. Em 2012, a "Gincana" foi desenvolvida a distância, sendo denominada "Gincana Virtual". Métodos: Equipes foram formadas e tarefas eram formuladas e trocadas entre elas, sendo avaliadas a construção da tarefa, factibilidade, pertinência de conteúdo e resposta. A gincana foi aplicada na modalidade a distância no primeiro semestre $2012(n=40)$ e presencial no segundo semestre do mesmo ano $(n=42)$. Foi respondido um questionário final de avaliação, considerando: Metodologia, Material, Tarefas e Considerações. Ainda foi avaliado o desempenho entre as modalidades. Resultados: A "Gincana" apresentou diferença significativa, sendo preferida pelos alunos na modalidade presencial. Conforme os comentários dos alunos, o uso do Fórum síncrono como forma de interação é inovador, porém pode dificultar a interação e o dinamismo da atividade. Por outro lado, incentiva a participação da turma, devido ao fato da EaD diminuir a exposição do aluno. Houve um melhor desempenho acadêmico, na média, na modalidade presencial. Conclusão: As gincanas promovem um aumento da atenção e da concentração dos estudantes por conta da competição, portanto, a ferramenta de interação da "Gincana Virtual" deve ser interativa e dinâmica, assim como ocorre no presencial.

Palavras-chave: aprendizagem; educação em farmácia; avaliação educacional; educação a distância; atenção farmacêutica.

\begin{abstract}
Introduction: The growth of distance education (DE) in Brazil has been increasingly intense, including in health courses. Objective: To do a comparative analysis between the modalities about assessment of activity and academic performance. "Scavenger Hunt" was developed in Distance Learning (DL) mode in 2012, called "Virtual Scavenger Hunt". Methods: The "Scavenger Hunt" was applied in DL mode in the first 2012 semester $(n=40)$ and face-to-face in the second semester $(n=42)$. The class was divided into two teams, for the first time the teams formulated tasks and the second time the tasks were exchanged between them. The students answered a final discipline assessment survey, considering Methodology, Collateral, Tasks and Considerations. Yet was assessed the academic performance between the two modes. Results: "Scavenger Hunt", as the Methodology and the Task was a significant difference, with a preference for the face-to-face kind. As for the comments of the students, the use of synchronous Forum as a means of interaction is innovative, but can cause confusion and slow to update, hindering the interaction and dynamism of activity. On the other hand, it makes the Class participation higher because DL reduces exposure of the student. There was a better academic performance, on average, in the face-to-face mode. Conclusion: The educational games promotes increasing students' attention and concentration because of the competition, so the interaction tool "Virtual Scavenger Hunt" should be interactive and dynamic, as well as in the face-to-face class.
\end{abstract}

Keywords: learning; education, pharmacy; educational measurement; education, distance; pharmaceutical care. 


\section{INTRODUÇÃO}

Segundo Anastasiou ${ }^{1}$, a Universidade está passando por um necessário movimento de transformação no processo de ensinamento, superando a metodologia tradicional associada à memorização e ao trabalho docente dirigido à explanação de conteúdos e retenção da atenção. Pela proposta atual, o processo de ensinamento deve se relacionar com a ação de apreender, com "as orientações pedagógicas não se referindo mais a passos a serem seguidos, mas a momentos a serem construído por sujeitos de ação". Isso significa utilizar metodologias de ensino que coloquem o aluno no centro do processo, como é princípio das metodologias ativas.

$\mathrm{Na}$ área da saúde, não é diferente, a educação dos profissionais da saúde ainda baseia-se, na sua maioria, em um modelo fragmentado do saber, desconsiderando as necessidades de atuação prática e as do Sistema Único de Saúde (SUS). Da mesma forma, as metodologias de ensino utilizadas na formação do profissional da saúde são as tradicionais, caracterizadas pela transferência de conhecimento do docente ao aluno, de forma tecnicista e desconsiderando o contexto cultural onde o aluno está inserido ${ }^{2}$.

Os jogos didáticos surgem como uma alternativa, pois incentivam o trabalho em equipe e a interação aluno-professor; auxiliam no desenvolvimento de raciocínio e habilidades; e facilitam o aprendizado de conceitos ${ }^{3}$. De acordo com Oliveira ${ }^{4}$, no jogo educacional, há algo que o jogador procura atingir e, nessa procura, ocorre o despertar do interesse que chama a atenção de quem dele participa, fazendo aflorar habilidades que muitas vezes estão escondidas, que leva o indivíduo a encontrar soluções aos diferentes problemas que encontram em diferentes situações durante o desenvolver de sua existência.

Apesar da estratégia de ensino baseada em jogos ser bastante consolidada na área da educação ${ }^{5}$, na área da Farmácia, o uso e pesquisa desta estratégia ainda é inovador. Quando comparado com métodos de ensino mais tradicionais, o aprendizado baseado em jogos promete uma maior motivação dos alunos, por ser uma forma de apresentar o conteúdo de uma maneira mais interativa. Estudos demonstram que o estímulo ao processo de aprendizagem não se dá apenas no domínio cognitivo, mas também nos domínios afetivos e psicomotores ${ }^{6,7}$. Por outro lado, algumas revisões sobre esse tema, na área médica, demonstram que as evidências muitas vezes são equivocadas e limitadas ${ }^{8-10}$. Estudo recente demonstrou que o método de aprendizado baseado em jogos é comparável ao método tradicional em geral e em ganhos de curto prazo, enquanto a aula tradicional ainda parece ser mais eficaz para melhorar a retenção de conhecimento de curto e longo prazos dos alunos ${ }^{11}$.

Um jogo educativo pode suportar discussões de alto nível que ajudam a melhorar a comunicação dos alunos, colaboração social e habilidades de pensamento crítico, todos os quais são habilidades essenciais para o Farmacêutico ${ }^{12,13}$. As evidências da eficácia pedagógica dos jogos, quando utilizados na área da saúde, ainda estão em questionamento, apesar de estudos demonstrarem suas vantagens ${ }^{14}$. Alguns estudos verificaram a utilidade dos jogos no contexto da educação farmacêutica ${ }^{15-26}$. Encontrou-se apenas uma revisão que resume e valida os resultados positivos associados com jogos educativos utilizados nas escolas de Farmácia ${ }^{27}$.

A disciplina de Atenção Farmacêutica II começou a fazer parte do currículo de Graduação da Faculdade de Farmácia da Universidade Federal do Rio Grande do Sul (UFRGS) no ano de 2008, e hoje utiliza a modalidade a distância no seu programa de ensino. Tendo em vista a recente inclusão do uso das tecnologias de informação e comunicação no ensino na área da saúde, poucos estudos foram encontrados investigando se esses recursos e as atividades a distância apontam diferenças no desempenho dos acadêmicos em relação à modalidade presencial. $\mathrm{O}$ objetivo deste estudo é realizar uma análise comparativa da atividade "Gincana" nas modalidades presencial e a distância. A "Gincana" foi utilizada desde 2008 na modalidade presencial, na disciplina de Atenção Farmacêutica II, do Curso de Farmácia. Em 2012, foi desenvolvida na modalidade a distância, sendo denominada "Gincana Virtual”. Os aspectos avaliados nesta comparação são: a percepção e satisfação dos estudantes em relação a cada aula e o desempenho do estudante entre as duas modalidades.

\section{MÉTODOS}

\section{Disciplina de Atenção Farmacêutica II}

O objetivo da disciplina é de proporcionar ao aluno o conhecimento básico para que possa atuar na Farmácia, promovendo o uso racional de medicamentos, por meio do desenvolvimento de habilidades para a busca, análise e utilização de informações, na dispensação de medicamentos e orientação farmacêutica de pacientes.

A disciplina é dividida em dezesseis aulas, sendo 5 do Módulo I e 11 do Módulo II. Diversas estratégias de ensino foram utilizadas na disciplina, como mapa conceitual, tutoriais de busca de informação, simulação de atendimento ao paciente, tribunal do júri. A estratégia da Gincana foi escolhida por proporcionar ao aluno vivenciar o trabalho em equipe e a criatividade no planejamento das tarefas, adicionalmente à consolidação do conteúdo trabalhado em aula.

Para realização deste estudo, a "Gincana” foi ofertada na modalidade a distância no primeiro semestre de 2012 e na modalidade presencial no segundo semestre do mesmo ano. Para a avaliação da atividade, utilizou-se os dados da disciplina referentes ao alunado e às avaliações da disciplina, nos semestres de 2012/01 e 2012/02.

\section{Descrição da Dinâmica}

A "Gincana” é uma das atividades avaliativas da disciplina de Atenção Farmacêutica II do Curso de Farmácia da Universidade Federal do Rio Grande do Sul desde 2008, após Reforma Curricular motivada pelas Diretrizes Curriculares editadas pelo Ministério da Educação, em fevereiro de $2002^{28}$. 
Primeiramente, a turma foi dividida em duas equipes e tinham que elaborar três tipos de tarefas cada (Jogo dos sete erros, Jogo de Adivinhação, Mímica e Palavras cruzadas). As tarefas elaboradas eram avaliadas pelos professores em relação à factibilidade de resolução das mesmas, ao tempo e ao conteúdo empregados. No segundo momento da aula, as tarefas eram trocadas e as equipes tinham o período da aula para realizá-la. A avaliação da atividade foi em relação às tarefas elaboradas, tarefas resolvidas e trabalho em equipe, sendo que a equipe que elaborasse e resolvesse primeiro as tarefas tinham uma pontuação maior. O tema da "Gincana" era sobre os conteúdos que haviam sido trabalhados no semestre: Atenção Farmacêutica (dispensação de medicamentos, orientação farmacêutica, seguimento farmacoterapêutico), Adesão ao Tratamento e Erros de Medicação.

\section{A "Gincana Virtual"}

A "Gincana Virtual” foi baseada na atividade desenvolvida presencialmente, utilizando-se a plataforma virtual Moodle ${ }^{29}$, um sistema de gerenciamento de cursos de aprendizagem de código aberto que possibilita educadores a criar comunidades de aprendizagem online eficazes. A ferramenta para as equipes se comunicarem, elaborarem e resolverem as tarefas foi o Fórum síncrono, ou seja, todos estavam online, para realizar as discussões. As equipes foram divididas em grupos fechados (somente a própria equipe tinha acesso às publicações). No primeiro momento, o fórum foi utilizado para o grupo compartilhar e registrar a elaboração das tarefas. Em seguida, a equipe postava, no espaço daquela aula, um documento com as tarefas elaboradas. As mesmas eram analisadas pelos professores, utilizando-se os mesmos critérios da atividade presencial, porém considerando-se agora a factibilidade de realização da tarefa no ambiente virtual. No segundo momento da aula, as equipes se reuniram no Fórum de Resolução das Tarefas e tinham o tempo de uma aula para realizar e postar as mesmas. A avaliação da "Gincana Virtual" seguiu os mesmos critérios da atividade presencial.

\section{Amostra}

A amostra foi composta por acadêmicos matriculados na $4^{\text {a }}$ etapa do Curso de Graduação em Farmácia, na disciplina de Atenção Farmacêutica II (FAR02017), nos semestres letivos de 2012/1 e 2012/2. A amostra foi por conveniência, de acordo com os matriculados em cada semestre. A gincana foi aplicada na modalidade a distância no primeiro semestre $2012(n=40)$ e presencial no segundo semestre do mesmo ano $(n=42)$. Os estudantes, independente do ambiente, tiveram as mesmas aulas, mesmos conteúdos, mesmos professores, e mesmos objetivos a serem atingidos na avaliação, sendo a única diferença entre os grupos a modalidade de ensino. Os alunos da disciplina passaram por avaliações de conhecimento e desempenho durante o semestre que incluíam os seguintes conteúdos: Módulo 1 - Exercício de avaliação de habilidades de construção de informação sobre medicamentos; Módulo 2 - Apresentação de base teórica para a realização de dispensação e orientação farmacêutica.

\section{Instrumentos}

Os instrumentos utilizados no estudo foram constituídos de dois questionários de avaliação da disciplina, sendo um aplicado no início do semestre, para identificar o perfil dos alunos e suas preferências e um segundo no final do semestre, sobre a avaliação das duas modalidades utilizadas e de cada aula. Os questionários foram estruturados com base nos pontos encontrados nos estudos comparativos entre modalidades a distância e presencial, pesquisados anteriormente em revisão bibliográfica.

- Questionário 1 - Perfil dos estudantes: O questionário aplicado no início da disciplina tinha como objetivo traçar o perfil demográfico dos estudantes e seu grau de inclusão digital, sendo estas as variáveis basais. Estas variáveis podem ser relacionadas com a satisfação do estudante em relação à modalidade a distância e a modalidade presencial e seu desempenho em ambas.

- Questionário 2 - Avaliação da Atividade: Para avaliar a atividade, os alunos responderam um segundo questionário de avaliação final da disciplina, no qual foi solicitada a avaliação de cada atividade realizada ao longo da disciplina. Nesta avaliação, os alunos preencheram questões sobre as aulas, avaliando especificamente a atividade da "Gincana", avaliando três critérios relevantes (Metodologia de aula, Material de apoio e Tarefas). A metodologia de aula seria a dinâmica da "Gincana", utilizando o fórum para discussão na modalidade a distância e encontro presencial na modalidade presencial. Os textos disponibilizados no ambiente da disciplina foram utilizados como material de apoio para embasar a discussão e realização da atividade. As tarefas referiam-se às participações dos alunos no fórum de discussão e a entrega das tarefas formuladas e realizadas.

Para a realização da avaliação desta atividade, os alunos tiveram que conceituar cada critério entre as seguintes escalas: péssima, ruim, boa, ótima e sem condições de opinar. Ainda, com o intuito de complementar a avaliação, havia um espaço para considerações gerais sobre a aula em questão, sendo esta parte do questionário analisada de forma qualitativa, utilizando-se a Análise de Conteúdo de $\operatorname{Bardin}^{30}$. Essa opção metodológica justifica-se pelo fato de a análise temática ser rápida e eficaz na condição de aplicar-se a discursos diretos (significações manifestas) e simples, como é o caso dos dados coletados no presente estudo. A análise de conteúdo é conceituada por Bardin ${ }^{30}$ como um conjunto de técnicas de análise das comunicações, visando obter, por procedimentos, sistemáticos e objetivos de descrição de conteúdo das mensagens, indicadores (quantitativos ou não) que permitam a inferência de conhecimentos relativos às condições de produção/recepção (variáveis inferidas) destas mensagens. Para $\operatorname{Bardin}^{30}$, a análise de conteúdo basicamente desdobra-se em três fases (pré-análise, exploração do material e tratamento dos resultados, inferência e interpretação), fases estas que foram utilizadas neste estudo como norteadoras da análise. Para realizar a análise qualitativa de 
conteúdo, utilizou-se o software NVivo, em que todas as respostas foram transcritas, analisadas e categorizadas e posteriormente interpretadas.

\section{Avaliação do desempenho acadêmico}

A fim de comparar o desempenho acadêmico dos estudantes quando ofertadas atividades presencias ou a distância, foi analisado o desempenho do aluno em cada semestre. Utilizou-se o artifício da nota para facilitar a comparação estatística em lugar de conceito. Normalmente, como norma da Universidade, a avaliação em pontos é traduzida em conceito. O desempenho dos alunos na Gincana foi determinado pela nota dada pelo professor a cada uma das equipes. Cada grupo recebeu uma nota considerando-se: trabalho realizado em grupo, capacidade de organização do grupo para a realização de tarefas, qualidade das tarefas elaboras, qualidade das respostas e pontualidade.

\section{Análise estatística}

A análise dos dados foi realizada usando a versão 17.0 do SPSS. Teste $t$ de Student, amostras teste $t$ pareado, análise ANOVA, correlação de Pearson e Mann Whitney foram utilizados nas comparações estatísticas do questionário 1. A análise não paramétrica de Mann Whitney $U$ foi conduzida para comparar as avaliações das aulas entre os dois semestres, referente às questões do questionário 2.

\section{RESULTADOS}

\section{Perfil dos estudantes matriculados no ano de 2012 na disciplina de Atenção Farmacêutica II}

O perfil dos alunos do ano de 2012 está apresentado na Tabela 1. Em relação à idade e sexo, não houve diferenças significativas entre as turmas avaliadas, sendo que a maioria da amostra era do sexo feminino, o que condiz com o perfil dos estudantes de Farmácia. A média de idade dos estudantes é de 23,9 anos, sendo todos os participantes pertencentes à mesma geração, tendo acesso à internet desde a sua adolescência, no mínimo. A maioria era solteira $(95,9 \%)$ e não possuía filhos $(98,6 \%)$. Quanto à ocupação, a maioria realiza estágio de iniciação científica com bolsa (57,7\%), estágio este realizado nos laboratórios da Faculdade de Farmácia ou nos demais campus da Universidade Federal do Rio Grande do Sul. A maioria dos estudantes prefere trabalhar em duplas $(53,5 \%)$, o que pode indicar que os alunos tendem a não ser individualistas, ou preferem dividir atribuições sem grandes responsabilidades individuais. As áreas de atuação que os estudantes mais pretendem atuar é a de Farmácia Industrial (20,6\%) e Análises clínicas (17,6\%), áreas caracterizadas pela utilização de procedimentos técnicos com rotina diária. Entretanto, 23,5\% dos estudantes não sabe que área pretende seguir sendo que áreas que integram conhecimentos técnicos e humanísticos, como Atenção
Farmacêutica, Assistência Farmacêutica e Saúde Pública não foram citadas pelos estudantes.

A maioria dos alunos (71,2\%) está satisfeita com o curso de Farmácia. A expectativa em relação à disciplina condiz em parte com os objetivos da mesma, sendo que a maioria considera os seguintes pontos como expectativa: aprofundar o seu conhecimento, aprender sobre dispensação e uso racional e conhecer mais sobre medicamentos.

\section{Avaliação da aula}

Todas as aulas foram avaliadas individualmente, quantitativamente, quanto à metodologia, materiais e tarefas, após foram comparados os resultados de cada aula em relação ao módulo a distância e presencial. Para comparar o resultado das duas modalidades, foi utilizado o teste de Mann-Whitney. A aula da "Gincana" apresentou diferença significativa entre as duas modalidades, sendo preferidos os métodos e tarefas das aulas presenciais (Tabela 2).

Em relação ao material da aula, que não houve diferença significativa entre os grupos, para as atividades a distância, textos de leitura obrigatória e complementar, materiais de revisão e espaços de dúvidas foram postados na plataforma Moodle; já na aula presencial, o material da aula disponível a eles foram livros de livre consulta.

\section{Considerações sobre a Aula}

A última questão da avaliação da aula era uma pergunta aberta em que 32 pessoas responderam (39\%). As respostas

Tabela 1: Perfil de estudantes da disciplina Atenção Farmacêutica II, nos semestres 1 e 2 do ano de 2012

\begin{tabular}{|c|c|c|}
\hline Variáveis & n & $\%$ \\
\hline \multicolumn{3}{|l|}{ Turmas } \\
\hline 2012/01 A & 18 & 22,0 \\
\hline 2012/01 B & 18 & 22,0 \\
\hline $2012 / 01$ C & 4 & 4,9 \\
\hline $2012 / 02 \mathrm{~A}$ & 15 & 18,3 \\
\hline 2012/02 B & 15 & 18,3 \\
\hline $2012 / 02$ C & 12 & 14,6 \\
\hline Total & 82 & \\
\hline \multicolumn{3}{|l|}{ Gênero } \\
\hline Feminino & 72 & 88,9 \\
\hline Masculino & 9 & 11,1 \\
\hline \multicolumn{3}{|l|}{ Idade } \\
\hline Média (anos) & 23,9 & \\
\hline \multicolumn{3}{|l|}{ Estado civil } \\
\hline Solteiro & 70 & 95,9 \\
\hline Casado & 3 & 4,1 \\
\hline \multicolumn{3}{|l|}{ Ocupação } \\
\hline Estagiário IC & 41 & 57,7 \\
\hline Empregado assalariado & 2 & 2,8 \\
\hline Profissional liberal & 1 & 1,4 \\
\hline Dona de casa & 1 & 1,4 \\
\hline Estagiário de extensão & 3 & 4,2 \\
\hline Estagiário voluntário & 4 & 5,6 \\
\hline Outras & 19 & 26,8 \\
\hline
\end{tabular}

IC: iniciação científica. 
foram transcritas na íntegra para o software NVivo na fase de recorte, em seguida foi realizada a classificação de cada trecho e a categorização, resultando na agregação em 5 categorias: Fórum como ferramenta; Interação entre os colegas; Comunicação confusa; Problemas relacionados à conexão e Preferência pelo presencial.

- Categoria 1 - Fórum como ferramenta: nesta categoria, os alunos apresentaram comentários a respeito do uso da ferramenta fórum na comunicação interna dos grupos e na comunicação entre os grupos e professores/monitores durante a atividade de "Gincana Virtual". O primeiro ponto levantado foi sobre a velocidade de troca de mensagens que não seria suficientemente rápida para garantir o dinamismo da atividade proposta. Cabe ressaltar que, nesta dinâmica da "Gincana Virtual", optou-se pelo uso do Fórum ao invés de uma ferramenta mais dinâmica como o chat, pois esse é de mais difícil acompanhamento. Outro ponto levantado foi o fato do fórum facilitar o contato com o professor, visto que as dúvidas que haviam eram resolvidas de forma coletiva e todos tomavam conhecimento das informações fornecidas.

Acho que o fórum tem uma atualização um pouco lenta para fazer esta atividade.

O fórum nessa atividade é bom, pois todos ficam conectados ao mesmo tempo e podem trocar informações e tirar dúvidas.

- Categoria 2 - Interação entre os Colegas: nessa categoria são agregados comentários a respeito da interação que a atividade gera, tanto na modalidade presencial, quanto a distância. Em relação à atividade presencial, os alunos comentaram que ela possibilita a interação entre os alunos, apesar de algumas confusões geradas pela dificuldade de dividirem tarefas. Já na modalidade a distância, fica evidente que aqueles alunos mais tímidos ficaram mais à vontade para participar e se expor.

Sou tímida, achei que não ia gostar da atividade, mas assim consegui trabalhar com os colegas.
A interação entre a turma foi boa (na Gincana presencial), apesar das pequenas confusões nas divisões das tarefas.

- Categoria 3 - Comunicação Confusa: essa categoria reúne comentários dos alunos sobre problemas de comunicação tanto na modalidade a distância, quanto na modalidade presencial. Na modalidade presencial eles relatam que é difícil dividir tarefas e os integrantes da equipe entenderem o papel de cada um.

Muita confusão, pessoas se metendo nas tarefas das outras.

Já a distância, verifica-se que também existiram problemas de comunicação, mas esses foram pautados mais pela adoção da ferramenta Fórum utilizada para a troca de mensagens e também pela postagem simultânea que acaba causando confusão.

Atividade simultânea com os demais colegas é confusa.

- Categoria 4 - Problemas relacionados à Conexão: essa categoria descreve os problemas relacionados às falhas de conexão apresentadas pelos alunos, durante a atividade de "Gincana Virtual", que acabou prejudicando o andamento da dinâmica.

Atividades em grupo e ter que estar todos conectados no mesmo horário é ruim.

A conexão caia bem na hora da discussão.

- Categoria 5 - Preferência pelo presencial: esta categoria agrega respostas dadas pelos alunos que realizaram a atividade na modalidade a distância, demonstraram uma preferência pela atividade presencial, principalmente pelos fatores do dinamismo, diversão e facilidade de aprendizado.

Presencial seria melhor, mais dinâmico, divertido e fácil de aprender.

Tabela 2: Avaliação da Aula 13 que utilizou como estratégia de ensino-aprendizagem a Gincana, nas modalidades presencial e a distância

\begin{tabular}{|c|c|c|c|c|c|c|c|}
\hline Modalidade & $\mathbf{n}$ & $\begin{array}{l}\text { Ranque das } \\
\text { médias }\end{array}$ & Soma dos Ranks & Mann-Whitney U & Wilcoxon W & z & $\begin{array}{l}\text { Asymp. Sig. } \\
\text { (2-tailed) }\end{array}$ \\
\hline \multicolumn{8}{|l|}{ Método } \\
\hline $\mathrm{EaD}$ & 34 & 26,84 & 912,50 & \multirow{3}{*}{317,5} & 912,5 & -3 & $0,004^{*}$ \\
\hline Presencial & 31 & 39,76 & 1232,50 & & & & \\
\hline Total & 65 & & & & & & \\
\hline \multicolumn{8}{|l|}{ Material } \\
\hline $\mathrm{EaD}$ & 34 & 28,81 & 979,50 & \multirow{3}{*}{384,5} & 979,5 & -2 & 0,050 \\
\hline Presencial & 31 & 37,60 & 1165,50 & & & & \\
\hline Total & 65 & & & & & & \\
\hline \multicolumn{8}{|l|}{ Tarefa } \\
\hline EaD & 34 & 27,06 & 920,00 & \multirow{3}{*}{325} & 920 & -3 & $0,005^{*}$ \\
\hline Presencial & 31 & 39,52 & 1225,00 & & & & \\
\hline Total & 65 & & & & & & \\
\hline
\end{tabular}

${ }^{*} \mathrm{p}<0,5$; significância determinada utilizando-se o Teste $t$ de Student; EaD: ensino a distância. 


\section{Desempenho do alunado}

A análise Bivariada Teste $t$ de Student (Tabela 3) revelou uma diferença significativa $(\mathrm{p}<0,05)$ entre os dois grupos comparados, Gincana Virtual e presencial, sendo a média dos desempenhos dos alunos na atividade da Gincana maior na modalidade presencial.

Mesmo tendo uma evidência de que o desempenho do aluno seja melhor na modalidade presencial, verificou-se que o desempenho dos alunos foi satisfatório em ambas as modalidades.

\section{DISCUSSÃO}

Jogos educacionais são considerados métodos de aprendizagem experiencial que podem contribuir de forma positiva para o aprendizado dos alunos ${ }^{31}$. A investigação nesta área explora o impacto destes jogos sobre a satisfação, o conhecimento, a mudança de atitude dos estudantes de Farmácia, e a participação dos $\operatorname{mesmos}^{32}$. No estudo atual, os estudantes demonstraram ter uma satisfação positiva em relação à atividade, porém preferiram aspectos como método e tarefa na modalidade presencial. Essa preferência pela modalidade presencial em alguns aspectos pode ser explicada quando se avalia as considerações qualitativas apontadas pelos estudantes que foram agrupadas pela Categoria 5, sobre a preferência pelo presencial. Conforme essa análise, verificou-se na fala dos alunos justificativas que levam a crer que a modalidade presencial seja mais fácil do que a distância, porque as interações são mais diretas e os processos de comunicação são mais efetivos. Outra hipótese que explica essa preferência pelo presencial é que, ao utilizar a plataforma Moodle e a ferramenta fórum para realizar a atividade a distância, o aluno tem que passar por essas duas barreiras de aprendizado, antes de realizar a atividade em si, algo que no presencial é direto, sem interferentes e outros aprendizados prévios, pois esse é o modelo conhecido. Essas barreiras relatadas poderiam ter sido superadas se houvesse uma inclusão digital inicial dos alunos no ambiente utilizado na disciplina.

Alguns jogos relatados como efetivos em outros estudos foram similares ao utilizados na "Gincana", como, por exemplo, ao jogo de Grady et al. ${ }^{21}$, que adotou Quem Quer Ser um Milionário, similar ao jogo de Adivinhações, que estava entre uma tarefa da "Gincana". Além disso, no estudo de Shah ${ }^{22}$, as palavras cruzadas foram encaradas de forma positiva quando utilizadas durante as aulas para reiniciar a atenção dos alunos. Uma característica levantada como positiva nos estudos envolvendo estudantes de Farmácia foi a possibilidade de interação gerada pelos jogos, fato que ficou evidente nos comentários dos alunos, agregados na Categoria 2: Interação entre os colegas. Conforme apontado no

Tabela 3: Comparação do desempenho dos estudantes entre a "Gincana Virtual" e a Gincana na modalidade presencial

\begin{tabular}{|l|c|c|c|}
\hline & $\mathbf{n}$ & Média (Desvio padrão) & Valor p \\
\hline Gincana EaD & 36 & $7,5167(0,41)$ & $0,000^{*}$ \\
\hline Gincana presencial & 35 & $8,8343(0,62)$ & \\
\hline
\end{tabular}

${ }^{*} \mathrm{p}<0,5$; significância determinada utilizando-se o Teste $t$ de Student; EaD: ensino a distância. estudo de Patel ${ }^{14}$, o ambiente com menos estresse proporcionado por um jogo educacional leva ao estudante a diminuir a ansiedade relacionada à graduação e proporciona um ambiente propício às interações. Ainda, no estudo atual, verificamos que na modalidade a distância essa interação é ainda maior nos casos de alunos que demonstram timidez na aula presencial, sendo relatado por alguns alunos que a "Gincana Virtual” incentivou sua participação.

Patel ${ }^{14}$ relata que, para os Jogos serem eficazes, eles devem compartilhar determinados critérios para garantir um impacto positivo na aprendizagem dos alunos. Primeiramente, seus objetivos e resultados educacionais esperados devem ser claramente definidos. Este fator da definição dos critérios pode ter prejudicado a clareza da atividade da "Gincana", visto que, na proposta da atividade, não foram deixados claros todos os critérios de avaliação exigidos, o que proporcionou que algumas pessoas participassem mais que as outras. Também é importante verificar que, segundo Marsh et al. ${ }^{32}$, o equilíbrio entre o entretenimento e componentes educacionais precisa ser considerado, pois quanto mais agradável um jogo educativo é, maior o interesse dos alunos em jogá-lo. Essa afirmação e característica importante de um jogo educacional pode ter influenciado na avaliação da "Gincana Virtual", pois, conforme apresentado nas categorias 3 e 4, os estudantes acharam a comunicação confusa com o uso dos fóruns e ainda levantaram problemas relacionado à conexão da internet. Esses dois parâmetros e interferentes podem ter influenciado nas avaliações negativas em relação à realização da dinâmica no ambiente virtual.

Uma das variáveis categorizadas em nossa análise foi a interação que os jogos educacionais geram, seja qual for a modalidade utilizada. Verifica-se que essa característica é relatada como positiva e contribui para o aprendizado. No estudo de Persky ${ }^{26}$, essa também foi uma vantagem descrita. Quanto ao desempenho dos estudantes ter sido maior na modalidade presencial, é possível inferir que nessa modalidade, como relatado pelos alunos, o envolvimento do aluno seja maior, por causa da adrenalina e competição. Lenz ${ }^{33}$ também observou que o aluno de farmácia teve um desempenho maior na modalidade a presencial, que pode ser explicado pelo fato do aluno já estar acostumado com essa modalidade.

Grady ${ }^{21}$, após realizar uma revisão sobre estudos de avaliação de jogos educacionais e seus impactos no desempenho dos estudantes de Farmácia, concluiu que, para se realizar uma investigação de qualidade, deve-se utilizar um desenho metodológico adequado para se chegar às conclusões firmes sobre os impactos dos jogos educacionais na área da Farmácia. Sendo assim, é importante evidenciar que o desempenho dos estudantes nesse estudo atual foi positivo nas duas modalidades utilizadas, e a atividade de gincana pode ser aplicada de forma efetiva quando o objetivo é a revisão dos conteúdos aplicados em aula. A opção pelo uso do fórum como recurso para apoiar a interação na modalidade a distância demonstrou-se adequada, porém critérios de participação e avaliação tem que ser claramente comunicados. A interação é um importante componente para o aprendizado e é bastante estimulada pelos jogos educacionais. 


\section{REFERÊNCIAS}

1. Anastasiou LGC. Ensinar, aprender, apreender e o processo de ensinagem. In: Anastasiou LGC, Alves LP. Processos de Ensinagem na Universidade. Joinvile: Univale; 2012; p. 17-40.

2. Mitre SM, Siqueira-Batista R, Girardi-de-Mendonça JM, Morais-Pinto NM, Meirelles CAB, Pinto-Porto C, et al. Metodologias ativas de ensino-aprendizagem na formação profissional em saúde: debates atuais. Ciênc Saúde Coletiva. 2008;13 (Suppl 2):2133-44.

http://dx.doi.org/10.1590/S1413-81232008000900018

3. Vygotsky L. A formação social da mente. São Paulo: Martins Fontes; 1989.

4. Oliveira N. Atividades de experimentação investigativas lúdicas no ensino de química: um estudo de caso. Tese (Doutorado) Universidade Federal de Goiás. Goiânia: 2009; p. 52.

5. Dohme V. Atividades lúdicas na educação: o caminho de tijolos amarelos do aprendizado. 4ed. Petrópolis: Vozes; 2008.

6. Mitchell A. The use of computer and video games for learning: a review of the literature. London: Learning and Skills Development Agency; 2004

7. Schwartzman R. Gaming serves as a model for improving learning Education. 1997;118(1):9-17.

8. Akl EA, Sackett K, Pretorius R, Erdley S, Bhoopathi PS, Mustafa $\mathrm{R}$, et al. Educational games for health professionals. Cochrane Database Syst Rev. 2008;23(1):CD006411. http://dx.doi.org/10.1002/14651858.CD006411.pub2

9. Blakely G, Skirton H, Cooper S, Allum P, Nelmes P. Educational gaming in the health sciences: systematic review. J Adv Nurs. 2009;65(2):259-69. http://dx.doi.org/10.1111/ j.1365-2648.2008.04843.x

10. Akl EA, Pretorius RW, Sackett K, Erdley WS, Bhoopathi PS, Alfarah $Z$, et al. The effect of educational games on medical students learning outcomes: A systematic review: BEME Guide no 14. Med Teach. 2010;32(1):16-27. http://dx.doi.org/10.3109/01421590903473969

11. Rondon S, Sassi FC, Furquim de Andrade CR. Computer game-based and traditional learning method: a comparison regarding students' knowledge retention. BMC Med Educ. 2013;13:30.

http://dx.doi.org/10.1186/1472-6920-13-30

12. Guillén-Nieto V, Aleson-Carbonell M. Serious games and learning effectiveness: the case of it's a deal! Comput Educ. 2012;58(1):435-48 http://dx.doi.org/10.1016/j.compedu.2011.07.015

13. Cavanagh M. Students'experiences of active engagement through cooperative learning activities in lectures. Active Learning Higher Educ. 2011;12(1):23-33. http://dx.doi.org/10.1177/1469787410387724

14. Patel J. Using game format in small group classes for pharmacotherapeutics case studies. Am J Pharm Educ. 2008;72(1):21.

15. Barclay SM, Jeffres MN, Bhakta R. Educational card games to teach pharmacotherapeutics in an advanced pharmacy practice experience. Am J Pharm Educ. 2011;75(2):33.

16. Oliver CH, Hurd PD, Beavers M, Gibbs E, Goeckner B, Miller K. Experiential learning about the elderly: the geriatric medication game. Am J Pharm Educ. 1995;59(2):155-8.
17. Evans S, Lombardo M, Belgeri M, Fontane P. The geriatric medication game in pharmacy education. Am J Pharm Educ. 2005;69(3):46

18. Chen AMH, Plake KS, Yehle KS, Kiersma ME. Impact of the geriatric medication game on pharmacy students 'attitudes toward older adults. Am J Pharm Educ. 2011;75(8):158. http://dx.doi.org/10.5688/ajpe758158

19. Kennedy DH, Fanning KD, Thornton PL. The Age Game: An interactive tool to supplement course material in a geriatrics elective. Am J Pharm Educ. 2004;68(5):115

20. Roche VF, Alsharif NZ, Ogunbadeniyi AM. Reinforcing the relevance of chemistry to the practice of pharmacy through the who wants to be a med chem millionaire? Learning game. Am J Pharm Educ. 2004;68(5):116.

21. Grady SE, Vest KM, Todd TJ. Student attitudes toward the use of games to promote learning in the large classroom setting. Curr Pharm Teach Learn. 2013;5(4):263-8. http://dx.doi.org/10.1016/j.cptl.2013.01.008

22. Shah S, Lynch LMJ, Macias-Moriarity LZ. Crossword puzzles as a tool to enhance learning about anti-ulcer agents. Am J Pharm Educ. 2010;74(7):117.

23. Rose TM. A board game to assist pharmacy students in learning metabolic pathways. Am J Pharm Educ. 2011;75(9):183. http://dx.doi.org/10.5688/ajpe759183

24. Sando KR, Elliott J, Stanton ML, Doty R. An educational tool for teaching medication history taking to pharmacy students. Am $J$ Pharm Educ. 2013;77(5):105. http://dx.doi.org/10.5688/ajpe775105

25. Tietze KJ. A bingo game motivates students to interact with course material. Am J Pharm Educ. 2007;71(4):79. http://dx.doi.org/10.5688/aj710479

26. Persky AM, Stegall-Zanation J, Dupuis RE. Students' perceptions of the incorporation of games into classroom instruction for basic and clinical pharmacokinetics. Am J Pharm Educ. 2007;71(2):21.

27. Aburahma MH, Mohamed HM. Educational games as a teaching tool in pharmacy curriculum. Am J Pharm Educ. 2015;79(4):59. http://dx.doi.org/10.5688/ajpe79459

28. Brasil. Ministério da Educação. Conselho Nacional de Educação. Resolução CNE/CES n ${ }^{\circ}$ 2, de 19 de fevereiro de 2002: Institul Diretrizes Curriculares Nacionais do Curso de Graduação em Farmácia. Diário Oficial da União. Brasília: MEC; 2002.

29. Sabbatini RME. Ambiente de ensino e aprendizagem via internet a plataforma moodle. Instituto EduMed; 2007.

30. Bardin L. Análise de conteúdo. Lisboa: Edições 70; 1977.

31. Graafland M, Schraagen JM, Schijven MP. Systematic review of serious games for medical education and surgical skills training. Br J Surg. 2012;99(10):1322-30. http://dx.doi.org/10.1002/bjs.8819

32. Marsh T, Nickole LZ, Klopfer E, Xuejin C, Haas J, Osterweil S. Fun and Learning: blending design and development dimensions in serious games through narrative and characters. Serious Games Edutainment Applic. 2011;273-88.

33. Lenz TL, Monaghan MS, Wilson AF, Tilleman JA, Jones RM, Hayes MM. Using performance-based assessments to evaluate parity between a campus and distance education pathway. Am J Pharm Educ. 2006;70(4):90. 